Chirurg 2014 · 85:544

DOI 10.1007/s00104-014-2778-y

Online publiziert: 15. Mai 2014

(c) Springer-Verlag Berlin Heidelberg 2014

J. Reibetanz - C.-T. Germer

Klinik für Allgemein-, Viszeral-, Gefäß- und Kinderchirurgie, Universitätsklinik Würzburg

\title{
Adjuvante Chemotherapie nach neoadjuvanter Radiochemotherapie beim Rektumkarzinom
}

\section{Ergebnisse}

\section{Hintergrund und Fragestellung}

Für das lokal fortgeschrittene Rektumkarzinom belegen randomisiert-kontrollierte Studien den günstigen Effekt der neoadjuvanten Radiochemotherapie (verglichen mit der alleinigen Radiotherapie) hinsichtlich der lokalen Tumorkontrolle, jedoch ohne Einfluss auf das Gesamtüberleben. Auch die adjuvante Chemotherapie als Teil der multimodalen Therapie des Rektumkarzinoms führt scheinbar nicht zu einer signifikanten Überlebensverlängerung [1]. Aktuell werden die 10-Jahres-Ergebnisse der EORTC-22921Studie zur Frage eines möglichen Langzeiteffekts der adjuvanten Chemotherapie auf des krankheitsfreie und Gesamtüberleben vorgestellt.

\section{Methode}

Die EORTC-22921-Studie randomisierte in einem „ $2 \times 2$ factorial design“ Patienten mit resektablen Rektumkarzinomen (T3 oder T4, M0) in einen der folgenden $4 \mathrm{Be}$ handlungsarme:

- präoperative Radiotherapie (RT),

- präoperative Radiochemotherapie (RCT),

- präoperative Radiotherapie und postoperative Chemotherapie (CT),

- präoperative Radiochemotherapie und postoperative Chemotherapie.

Primärer Endpunkt war das Gesamtüberleben, sekundäre Endpunkte wa- ren krankheitsfreies Überleben, Lokalrezidivrate, Fernmetastasierung, Toxizität, Downstaging und pathologisches Komplettansprechen.

Diskussion und Fazit

des Reviewers

Die 10-Jahres-Ergebnisse der EORTC22921-Studie zeigen eindrücklich, dass die adjuvante Fluorouracil(5-FU)-basierte Chemotherapie nach präoperativer Radio(chemo)therapie ohne Einfluss auf das Gesamt- oder krankheitsfreie Überleben bleibt. Dieser mangelnde Einfluss lässt sich z. T. dadurch erklären, dass nur ein Teil der Patienten die adjuvante Chemotherapie tatsächlich erhält (bei EORTC 22921: 43\%) oder die aktuellen Regime (4 Zyklen 5-FU) vergleichsweise ineffektiv sind. In jedem Fall sollte eine generelle (leitliniengerechte) Empfehlung zur adjuvanten Chemotherapie nach vorangegangener Radiochemotherapie kritisch hinterfragt werden, da sie mutmaßlich keinen signifikanten Überlebensvorteil bietet. Dagegen scheint nach alleiniger präoperativer Radiotherapie eine adjuvante Chemotherapie das Lokalrezidivrisiko noch günstig beeinflussen zu können.

[KI] 47,0-56,4) für adjuvante Therapie vs. $48,4 \%$ (95\%-KI 43,6-53,0) für Surveillance (Hazard ratio [HR] 0,91; 95\%-KI 0,771,09, $\mathrm{p}=0,32)$. Krankheitsfreies Überleben: $47,0 \%$ (95\%-KI 42,2-51,6) für adjuvante Therapie vs. 43,7\% (95\%-KI 39,1-48,2) für Surveillance (HR 0,91; 95\%-KI 0,77-1,08, $\mathrm{p}=0,29$ ). Erneut bestätigte sich der günstige Effekt der kombinierten Radiochemotherapie gegenüber der alleinigen Radiotherapie hinsichtlich der Lokalrezidivrate $(\mathrm{p}=0,0017)$, wobei der Zeitpunkt der applizierten Chemotherapie (neoadjuvant als RCT oder adjuvant nach RT/RCT) ohne Einfluss blieb. Langzeitnebenwirkungen/Toxizitäten waren für alle $4 \mathrm{Be}$ handlungsarme vergleichbar $(p=0,22)$.

\section{Korrespondenzadresse}

Prof. Dr. C.-T. Germer

Klinik für Allgemein-, Viszeral-, Gefäß- und Kinderchirurgie, Universitätsklinik Würzburg, Oberdürrbacher Str. 6, 97080 Würzburg germer_c@ukw.de

Interessenkonflikt. J. Reibetanz und C.-T. Germer geben an, dass kein Interessenkonflikt besteht.

\section{Literatur}

1. Bosset JF, Collette L, Calais G et al (2006) Chemotherapy with preoperative radiotherapy with rectal cancer. N Engl J Med 355:1114-1123 